\title{
SOLVING AN OPEN PROBLEM ABOUT THE G-DRAZIN PARTIAL ORDER*
}

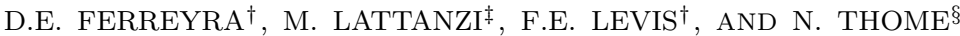

\begin{abstract}
G-Drazin inverses and the G-Drazin partial order for square matrices have been both recently introduced by Wang and Liu. They proved the following implication: If $A$ is below $B$ under the G-Drazin partial order, then any G-Drazin inverse of $B$ is also a G-Drazin inverse of $A$. However, this necessary condition could not be stated as a characterization and the validity (or not) of the converse implication was posed as an open problem. In this paper, this problem is completely solved. It is obtained that the converse, in general, is false, and a form to construct counterexamples is provided. It is also proved that the converse holds under an additional condition (which is also necessary) as well as for some special cases of matrices.
\end{abstract}

Key words. G-Drazin inverse, Space pre-order, Minus partial order, G-Drazin partial order.

AMS subject classifications. 15A09, 06A06.

1. Introduction and background. Generalized inverses of matrices are very useful in various applications such as Markov chains [3], chemical equations [14], robotics [8], coding theory [18], etc. In particular, the Drazin inverse has proved helpful in analyzing Markov chains, difference equations, differential equations, and iterative numerical methods, among other things. Taking into account its importance, many computational techniques were developed to calculate this generalized inverse [2, 17]. Campbell and Meyer considered some modifications to the classical Drazin inverse by introducing the concept of weak Drazin inverse [4]. Furthermore, generalized inverses play an important role in the study of matrix partial orders, as we can see in [13]. Interesting applications of partial orders were investigated, for example, in $[1,6,7,15]$.

Let $\mathbb{C}^{m \times n}$ be the set of $m \times n$ complex matrices. For $A \in \mathbb{C}^{m \times n}$, let $A^{*}, A^{T}, A^{-1}, \operatorname{rk}(A)$, and $\mathcal{R}(A)$ denote the conjugate transpose, the transpose, the inverse $(m=n)$, the rank, and the range space of $A$, respectively. Moreover, $I_{m}$ stands for the $m \times m$ identity matrix and $0_{m \times n}$ denotes the $m \times n$ zero matrix. If the size is clear from the context, it will be directly denoted by $I$ or 0 .

For $A \in \mathbb{C}^{m \times n}$, a matrix $X \in \mathbb{C}^{n \times m}$ that satisfies the equation $A X A=A$ is called an $\{1\}$-inverse of $A$, and it is denoted by $A^{-}$. The symbol $A\{1\}$ denotes the set of all $\{1\}$-inverses of $A$. For $A \in \mathbb{C}^{n \times n}$, the index of $A$ is the smallest nonnegative integer $k$ such that $\mathcal{R}\left(A^{k}\right)=\mathcal{R}\left(A^{k+1}\right)$, and is denoted by $k=i n d(A)$. For a given $A \in \mathbb{C}^{n \times n}$ with $k=\operatorname{ind}(A)$, a matrix $X \in \mathbb{C}^{n \times n}$ satisfying

$$
A X A=A, \quad X A^{k+1}=A^{k}, \quad \text { and } \quad A^{k+1} X=A^{k},
$$

${ }^{*}$ Received by the editors on September 11, 2019. Accepted for publication on December 4, 2019. Handling Editor: Oskar Maria Baksalary. Corresponding Author: Néstor Thome.

${ }^{\dagger}$ Universidad Nacional de Río Cuarto, CONICET, FCEFQyN, RN 36 KM 601, 5800 Río Cuarto, Córdoba, Argentina (deferreyra@exa.unrc.edu.ar, flevis@exa.unrc.edu.ar). Partially supported by Universidad Nacional de Río Cuarto (Grant PPI 18/C472), CONICET (Grant PIP 112-201501-00433CO), and by ANPCyT (Grant PICT 2018-03492).

${ }^{\ddagger}$ Universidad Nacional de La Pampa, FCEyN, Uruguay 151, 6300 Santa Rosa, La Pampa, Argentina (mblatt@exactas.unlpam.edu.ar). Partially supported by Universidad Nacional de La Pampa, Facultad de Ingeniería (Grant Resol. Nro. 155/14).

§Instituto Universitario de Matemática Multidisciplinar, Universitat Politècnica de València, 46022 Valencia, Spain (njthome@mat.upv.es). Partially supported by Ministerio de Economía, Industria y Competitividad of Spain (Grant Red de Excelencia MTM2017-90682-REDT), and by Universidad Nacional del Sur of Argentina (Grant 24/L108). 
is called a $G$-Drazin inverse of $A$. In general, there exists not only one matrix $X$ satisfying (1.1). The symbol $A\{G D\}$ stands for the set of all G-Drazin inverses of $A$; an element of this set is denoted by $A^{G D}$. Recently, in [5] the authors proved that the set of the matrix equations given in (1.1) is equivalent to the more simplified set of matrix equations given by $A X A=A$ and $A^{k} X=X A^{k}$.

Note that, by [13, Remark 2.2.24], the core-nilpotent decomposition of a matrix $A \in \mathbb{C}^{n \times n}$ of index $k$ can be stated as

$$
A=P\left[\begin{array}{cc}
\Sigma & 0 \\
0 & N
\end{array}\right] P^{-1}
$$

where $P \in \mathbb{C}^{n \times n}$ and $\Sigma \in \mathbb{C}^{t \times t}$ are both nonsingular matrices, and $N \in \mathbb{C}^{(n-t) \times(n-t)}$ is nilpotent with index $k$ and $t=\operatorname{rk}\left(A^{k}\right)$. In [16], the authors show that if $A$ is written as in (1.2), then every G-Drazin inverse of $A$ is given by

$$
A^{G D}=P\left[\begin{array}{cc}
\Sigma^{-1} & 0 \\
0 & N^{-}
\end{array}\right] P^{-1}, \quad \text { where } N^{-} \in N\{1\}
$$

Notice that the set of all matrices $N^{-} \in N\{1\}$ was investigated in [10, Theorem 2.1].

We also observe that if $A$ is nonsingular then $A\{G D\}=\left\{A^{-1}\right\}$. Moreover, the inclusion $A\{G D\} \subseteq A\{1\}$ always holds, while $A\{G D\}=A\{1\}$ is true whenever $A$ is a nilpotent matrix. Since $\operatorname{ind}(A)=\operatorname{ind}\left(A^{*}\right)$ and $\left(A^{*}\right)^{k}=\left(A^{k}\right)^{*}$ we have that

$$
X \in A\{G D\} \quad \text { if and only if } \quad X^{*} \in A^{*}\{G D\} .
$$

The following binary relations are well known. Let $A, B \in \mathbb{C}^{m \times n}$. The minus partial order and the space pre-order, denoted by $\leq^{-}$and $\preceq^{s}$, respectively, are given as follows (see [13]):

$$
\begin{aligned}
A \leq^{-} B & \Longleftrightarrow A^{-} A=A^{-} B \text { and } A A^{-}=B A^{-}, \text {for some } A^{-} \in A\{1\}, \\
A \preceq^{s} B & \Longleftrightarrow \mathcal{R}(A) \subseteq \mathcal{R}(B) \text { and } \mathcal{R}\left(A^{*}\right) \subseteq \mathcal{R}\left(B^{*}\right) \\
& \Longleftrightarrow\left(I-B B^{-}\right) A=0 \text { and } A\left(I-B^{-} B\right)=0, \text { for all } B^{-} \in B\{1\} .
\end{aligned}
$$

Recently, the G-Drazin partial order was defined by H. Wang and X. Liu as follows.

Definition 1.1. [16, Definition 3.1] Let $A, B \in \mathbb{C}^{n \times n}$. The matrix $A$ is said to be below the matrix $B$ under the G-Drazin partial order if there exist G-Drazin inverses $A_{G D}^{-}$and $A_{\bar{G}}^{\overline{\bar{D}} D}$ of $A$ such that $A_{G D}^{-} A=$ $A_{G D}^{-} B$ and $A A_{\bar{G} D}^{\bar{B}}=B A_{\bar{G} D}^{\bar{E}}$, and it is denoted by $A \leq^{G D} B$.

Although Definition 1.1 also can be given with only one G-Drazin inverse of $A$ (i.e., with $A_{G D}^{-}=A_{G D}^{\overline{\bar{C}}}$ ), our main result requires this version of the definition in order to be stated.

In [16, Theorem 3.4], it was proved that $A \leq^{G D} B$ implies $A \leq^{-} B$. Also, it is well known that the space pre-order can be derived from the minus partial order. Accordingly,

$$
A \leq^{G D} B \quad \Longrightarrow A \leq^{-} B \quad \Longrightarrow \quad A \preceq^{s} B .
$$

For more properties concerning these matrix partial orders we refer the reader to $[5,12,13]$. 
We recall that the classical matrix partial orders have the following general definition: $A \leq \leq^{\mathcal{G}} B$ iff $A^{\mathcal{G}} A=A^{\mathcal{G}} B$ and $A A^{\mathcal{G}}=B A^{\mathcal{G}}$, where $A^{\mathcal{G}}$ is a $\{\mathcal{G}\}$-inverse of $A$. For $A \in \mathbb{C}^{m \times n}$, we state the notations of the sets

$$
\begin{aligned}
A\{1,3\} & =\left\{X \in \mathbb{C}^{n \times m}: A X A=A,(A X)^{*}=A X\right\}, \\
A\{1,4\} & =\left\{X \in \mathbb{C}^{n \times m}: A X A=A,(X A)^{*}=X A\right\}, \\
A\{-, \operatorname{com}\} & =\left\{X \in \mathbb{C}^{n \times n}: A X A=A, A X=X A\right\}, \quad \text { for } m=n, \\
A\{-, c\} & =\left\{X \in \mathbb{C}^{n \times n}: X A=A^{\#} A\right\}, \quad \text { for } m=n .
\end{aligned}
$$

Now, very important characterizations for some partial orders are stated as follows [11, 12, 13]:

- (minus partial order) $A \leq^{-} B \Longleftrightarrow B\{1\} \subseteq A\{1\}$,

- (star partial order) $A \leq^{*} B \Longleftrightarrow B\{1,3\} \subseteq A\{1,3\}$ and $B\{1,4\} \subseteq A\{1,4\}$,

- (sharp partial order) $A \leq{ }^{\#} B \Longleftrightarrow B\{-, \operatorname{com}\} \subseteq A\{-, \operatorname{com}\}$,

- (core partial order) $A \leq \notin B \Longleftrightarrow B\{1,3\} \subseteq A\{1,3\}$ and $B\{-, c\} \subseteq A\{-, c\}$,

and so on.

In this paper, we study G-Drazin inverses, that are a particular case of weak Drazin inverses, and the G-Drazin partial order, both recently defined by H. Wang and X. Liu in [16]. The authors proved [16, Theorem 3.2] the following implication: if $A$ is below $B$ under the G-Drazin partial order then any G-Drazin inverse of $B$ is also a G-Drazin inverse of $A$. However, this necessary condition could not be stated as a characterization and the validity (or not) of the converse implication was posed as an open problem. By using recent results proved by the authors in [9], we are in position to solve completely this problem.

This paper is organized as follows. Section 2 shows that, in general, the aforementioned implication is false. Also, we prove that it holds under an additional condition as well as for some special cases of matrices. Section 3 yields an algorithm to construct numerical examples for any two square matrices of order $n, n \geq 3$, where the converse implication is false.

2. On the partial order based on G-Drazin inverses. The main goal of this section is to give a solution to the aforementioned open problem. In order to do that, we give some properties and provide new characterizations of the G-Drazin partial order that allow us to tackle the problem.

Next, we give some relationships between G-Drazin partial order, minus partial order, and Drazin preorder.

Proposition 2.1. Let $A, B \in \mathbb{C}^{n \times n}$. If $A \leq G D B$, then the following properties hold:

(i) $A^{D} \leq G D B^{D}$,

(ii) $A^{D} \leq^{-} B^{D}$ and $A^{D} \preceq^{D} B^{D}$,

(iii) $A^{D} \leq G D Z$, for any $Z \in B\{G D\}$.

Proof. The three properties are clear for $A=0$. Assume that $0 \neq A \leq G D B$ holds, let $k=i n d(A)$, and consider that the core-nilpotent decomposition of $A$ is written as in (1.2). Then, by [16, Theorem 3.1], we have

$$
B=P\left[\begin{array}{cc}
\Sigma & 0 \\
0 & B_{4}
\end{array}\right] P^{-1}, \quad \text { where } N \leq^{-} B_{4}
$$


(i) Since

$$
A^{D}=P\left[\begin{array}{cc}
\Sigma^{-1} & 0 \\
0 & 0
\end{array}\right] P^{-1} \quad \text { and } \quad B^{D}=P\left[\begin{array}{cc}
\Sigma^{-1} & 0 \\
0 & B_{4}^{D}
\end{array}\right] P^{-1} \quad(\text { see }[16, \text { p. 242]), }
$$

from $0 \leq^{-} B_{4}^{D}$ and [16, Theorem 3.1] we have that $A^{D} \leq^{G D} B^{D}$.

(ii) It is an immediate consequence of (i) and [16, Theorem 3.4].

(iii) From [5, Corollary 3.1], we have that $Z \in B\{G D\}$ if and only if

$$
Z=P\left[\begin{array}{cc}
\Sigma^{-1} & 0 \\
0 & Z_{4}
\end{array}\right] P^{-1} \quad \text { with } Z_{4} \in B_{4}\{G D\}
$$

Thus, by [16, Theorem 3.1], it is clear that $A^{D} \leq^{G D} Z$, for any $Z \in B\{G D\}$, because $0 \leq^{-} Z_{4}$.

Before stating the main theorem, we need the following auxiliary results.

Theorem 2.2. [9, Theorem 3.8] Let $A, B \in \mathbb{C}^{n \times n}$ be such that $B\{G D\} \subseteq A\{G D\}$. Let $k=\operatorname{ind}(A)$ and $\ell=\operatorname{ind}(B)$. Then the following properties hold:

(i) $\mathcal{R}(A) \subseteq \mathcal{R}(B)$ or $\mathcal{R}\left(A^{*}\right) \subseteq \mathcal{R}\left(B^{*}\right)$,

(ii) $\mathcal{R}\left(A^{k}\right) \subseteq \mathcal{R}\left(B^{\ell}\right)$ and $\mathcal{R}\left(\left(A^{k}\right)^{*}\right) \subseteq \mathcal{R}\left(\left(B^{\ell}\right)^{*}\right)$.

Lemma 2.3. Let $A, B \in \mathbb{C}^{n \times n}$ be such that $B\{G D\} \subseteq A\{G D\}$. Let $k=\operatorname{ind}(A)$ and $\ell=\operatorname{ind}(B)$. Then the following properties hold:

(i) $A^{k} \preceq^{s} B^{\ell}$,

(ii) $A \leq^{-} B$ if one of the following cases hold: (a) $\ell=0$, (b) $B^{\ell}=0$ for $\ell \geq 1$, (c) $k=0$,

(iii) $A \preceq^{s} B$ if $k=1$.

Proof. If $A=0$, the results are immediate. We may assume $A \neq 0$.

(i) It follows clearly from Theorem 2.2 (ii).

(ii) We consider the following three cases:

(a) If $\ell=0$, it is clear that $\left\{B^{-1}\right\}=B\{G D\} \subseteq A\{G D\}$, and so $A B^{-1} A=A$. Now, we have $B\{1\} \subseteq A\{1\}$, or equivalently $A \leq^{-} B$.

(b) If $B^{\ell}=0$ for $\ell \geq 1$, from (i) we see that $\mathcal{R}\left(A^{k}\right) \subseteq \mathcal{R}\left(B^{\ell}\right)=\{0\}$ gives $A^{k}=0$. Therefore, $A$ and $B$ are nilpotent matrices, and consequently, $B\{G D\}=B\{1\}$ and $A\{G D\}=A\{1\}$ hold. Thus, $B\{1\} \subseteq A\{1\}$, or equivalently, $A \leq^{-} B$.

(c) If $k=0$, by (i), we have $\mathbb{C}^{n}=\mathcal{R}\left(A^{k}\right) \subseteq \mathcal{R}\left(B^{\ell}\right)$, that is $B$ is nonsingular, and hence, $\ell=0$. From (a), we obtain $A \leq^{-} B$.

(iii) From (i), we get that $A \preceq^{s} B^{\ell}$ holds provided that $k=1$. Now, the conclusion follows directly from definition.

In [16, Theorem 3.2], the authors proved that

$$
A \leq{ }^{G D} B \text { implies } B\{G D\} \subseteq A\{G D\} .
$$


However, the issue of proving or disproving the converse implication was left as an open problem. Actually, it has been not easy to find the solution that we present in what follows due to the restricted set of matrices in which the result is not valid. Next, we give an additional condition in order that the converse in (2.6) becomes true and then we get the desired characterization.

TheOrem 2.4. Let $A, B \in \mathbb{C}^{n \times n}$. Then, the following conditions are equivalent:

(i) $A \leq G D B$

(ii) $B\{G D\} \subseteq A\{G D\}$ and $A \preceq^{s} B$.

Proof. $[(\mathrm{i}) \Rightarrow(\mathrm{ii})]$ Supposing that $A \leq{ }^{G D} B$ is true, the condition $B\{G D\} \subseteq A\{G D\}$ was proved in [16, Theorem 3.2] and the condition $A \preceq s B$ follows from (1.5).

$[($ ii $) \Rightarrow(\mathrm{i})]$ Let $k=\operatorname{ind}(A)$. Assuming that $B\{G D\} \subseteq A\{G D\}$ holds, we have $A Z A=A$ and $A^{k} Z=Z A^{k}$ for any $Z \in B\{G D\}$. Now, we fix $A^{G D} \in A\{G D\}$ and $B^{G D} \in B\{G D\}$. Let us consider the matrices $A_{G D}^{-}:=A^{G D} A B^{G D}$ and $A_{\bar{G}} \overline{\bar{S}}^{\mathrm{G}}:=B^{G D} A A^{G D}$. From

$$
A A_{G D}^{-} A=A A^{G D}\left(A B^{G D} A\right)=A A^{G D} A=A
$$

and

$$
A^{k} A_{G D}^{-}=A^{k} A^{G D} A B^{G D}=A^{G D} A A^{k} B^{G D}=A^{G D} A B^{G D} A^{k}=A_{G D}^{-} A^{k}
$$

we have that $A_{G D}^{-}$is a G-Drazin inverse of $A$. Similarly, $A_{G D} \in A\{G D\}$. On the other hand, since $A \preceq^{s} B$ we have $\mathcal{R}(A) \subseteq \mathcal{R}(B)$ and $\mathcal{R}\left(A^{*}\right) \subseteq \mathcal{R}\left(B^{*}\right)$. The second inclusion implies that there exists a matrix $Y$ such that $A=Y B$. Therefore,

$$
\begin{aligned}
A_{G D}^{-} A & =A^{G D}\left(A B^{G D} A\right)=A^{G D} A=A^{G D} Y B \\
& =A^{G D} Y\left(B B^{G D} B\right)=A^{G D} A B^{G D} B=A_{G D}^{-} B .
\end{aligned}
$$

Similarly, from $\mathcal{R}(A) \subseteq \mathcal{R}(B)$ we obtain $A=B X$ for some matrix $X$. Accordingly,

$$
\begin{aligned}
A A_{\bar{G}}^{\overline{\bar{F}}} & =\left(A B^{G D} A\right) A^{G D}=A A^{G D}=B X A^{G D} \\
& =\left(B B^{G D} B\right) X A^{G D}=B B^{G D} A A^{G D}=B A_{G D}^{\overline{\bar{G}}} .
\end{aligned}
$$

Finally, from (2.7), (2.8), and Definition 1.1, we arrive at $A \leq{ }^{G D} B$.

Next, we prove that the converse of (2.6) holds for some special cases.

Theorem 2.5. Let $A, B \in \mathbb{C}^{n \times n}$ be such that $k=\operatorname{ind}(A)$ and $\ell=\operatorname{ind}(B)$. Consider the following statements:

(i) $A \leq G D B$

(ii) $B\{G D\} \subseteq A\{G D\}$.

Then, (i) and (ii) are equivalent if one of the following conditions holds: (a) $k \leq 1,(b) \ell=0,(c) B^{\ell}=0$ for $\ell \geq 1$, (d) $r k\left(A^{k}\right)=\operatorname{rk}\left(B^{\ell}\right)>0$ for $k>1$ and $\ell \geq 1$.

Proof. $[(\mathrm{i}) \Rightarrow($ ii)] It was proved in [16, Theorem 3.2].

$[(i i) \Rightarrow(\mathrm{i})]$ If condition (a) holds, from Lemma 2.3 and (1.5) we have $A \preceq{ }^{s} B$. A similar reasoning is valid if either (b) or (c) hold. Therefore, the inequality $A \leq{ }^{G D} B$ follows from Theorem 2.4. 
Now, we assume that condition (d) holds. Let $B=P\left[\begin{array}{cc}\Sigma & 0 \\ 0 & N\end{array}\right] P^{-1}$ be the core-nilpotent decomposition of $B$, where $P \in \mathbb{C}^{n \times n}, \Sigma \in \mathbb{C}^{t \times t}$ are both nonsingular matrices, and $N \in \mathbb{C}^{(n-t) \times(n-t)}$ is nilpotent with index $\ell$. Thus, $\operatorname{rk}\left(B^{\ell}\right)=t$. Then

$$
B^{\ell}=P\left[\begin{array}{cc}
\Sigma^{\ell} & 0 \\
0 & 0
\end{array}\right] P^{-1} \quad \text { and } \quad B^{G D}=P\left[\begin{array}{cc}
\Sigma^{-1} & 0 \\
0 & N^{-}
\end{array}\right] P^{-1}
$$

with $N^{-} \in N\{1\}$. By Lemma $2.3(\mathrm{i})$ and [13, Theorem 3.2.10], we get $A^{k}=P\left[\begin{array}{cc}Z & 0 \\ 0 & 0\end{array}\right] P^{-1}$, with $Z \in$ $\mathbb{C}^{t \times t}$ and $\operatorname{ind}(Z) \leq 1$. Notice that $Z \in \mathbb{C}^{t \times t}$ is nonsingular, because $\operatorname{rk}(Z)=\operatorname{rk}\left(A^{k}\right)=t$. We partition $A=P\left[\begin{array}{ll}A_{1} & A_{2} \\ A_{3} & A_{4}\end{array}\right] P^{-1}$, accordingly to the sizes of the blocks of $B$.

As $B\{G D\} \subseteq A\{G D\}$, we have that $A B^{G D} A=A$ implies $\left(A B^{G D}\right) A^{k}=A^{k}$, and hence,

$$
P\left[\begin{array}{cc}
A_{1} \Sigma^{-1} Z & 0 \\
A_{3} \Sigma^{-1} Z & 0
\end{array}\right] P^{-1}=P\left[\begin{array}{cc}
Z \Sigma^{-1} A_{1} & Z \Sigma^{-1} A_{2} \\
0 & 0
\end{array}\right] P^{-1}=P\left[\begin{array}{cc}
Z & 0 \\
0 & 0
\end{array}\right] P^{-1} .
$$

Consequently, $A=P\left[\begin{array}{cc}\Sigma & 0 \\ 0 & A_{4}\end{array}\right] P^{-1}$ with $A_{4}^{k}=0$. Since $A B^{G D} A=A$, we see that $A_{4} N^{-} A_{4}=A_{4}$, i.e., $N^{-} \in A_{4}\{1\}$. The arbitrariness of $N^{-}$in the general expression of $B^{G D}$ implies $N\{1\} \subseteq A_{4}\{1\}$, and thus, $A_{4} \leq^{-} N$. Therefore, $A \leq{ }^{G D} B$ follows from [16, Theorem 3.4].

We would like to highlight that Theorem 2.5 provides us with several situations where the converse of (2.6) holds. This issue gives an idea of the difficulty offered in order to find the counterexample and it points the way where the effort have to be put to search for it. We are now in a position to show that, in general, the converse of (2.6) is false.

Example 2.6. Consider the matrices

$$
A=\left[\begin{array}{llll}
0 & 1 & 1 & 0 \\
0 & 0 & 0 & 0 \\
0 & 0 & 0 & 0 \\
0 & 0 & 0 & 0
\end{array}\right] \text { and } B=\left[\begin{array}{cccc}
0 & 1 & 0 & 0 \\
1 & 1 & 0 & 0 \\
0 & 0 & 0 & 1 \\
0 & 0 & 0 & 0
\end{array}\right]
$$

Clearly, $\operatorname{ind}(A)=\operatorname{ind}(B)=2$. Denoting $\Sigma=\left[\begin{array}{ll}0 & 1 \\ 1 & 1\end{array}\right]$ and $N=\left[\begin{array}{ll}0 & 1 \\ 0 & 0\end{array}\right]$ we have that

$$
B=\left[\begin{array}{cc}
\Sigma & 0 \\
0 & N
\end{array}\right] \text { with } N^{2}=0
$$

From (1.3) we get

$$
B^{G D}=\left[\begin{array}{cc}
\Sigma^{-1} & 0 \\
0 & N^{-}
\end{array}\right]
$$


where $\Sigma^{-1}=\left[\begin{array}{rr}-1 & 1 \\ 1 & 0\end{array}\right]$ and $N^{-}=\left[\begin{array}{ll}a & b \\ 1 & c\end{array}\right]$ with $a, b, c \in \mathbb{C}$. Also, we consider

$$
B^{-}=\left[\begin{array}{rrrr}
-1 & 1 & 0 & 0 \\
1 & 0 & 0 & 0 \\
0 & 0 & 0 & 0 \\
0 & 0 & 1 & 0
\end{array}\right] \in B\{1\}
$$

A simple computation shows that $A^{2}=0$ and, moreover, $B\{G D\} \subseteq A\{G D\}=A\{1\}$ holds. However, $A\left(I-B^{-} B\right) \neq 0$, that is, $\mathcal{R}\left(A^{*}\right) \not \mathcal{R}\left(B^{*}\right)$, and hence, $A \swarrow^{s} B$. In consequence, from (1.5) we have $A \not \mathbb{L}^{G D} B$.

The construction of the counterexample given above has been strongly based on the following Theorem 2.10. Before that, we need the following results.

TheOrem 2.7. [9, Theorem 3.2] Let $A \in \mathbb{C}^{n \times n}$ be a matrix of index $k$ and let $A^{G D}$ be a fixed G-Drazin inverse of $A$. Then the set of all G-Drazin inverses of $A$ is given by

$$
A\{G D\}=\left\{A^{G D}+\left(I-P_{A^{k}}\right) U\left(I-P_{A}\right)+\left(I-Q_{A}\right) V\left(I-P_{A^{k}}\right): U, V \text { are arbitrary }\right\},
$$

where $P_{A}=A A^{G D}, Q_{A}=A^{G D} A$, and $P_{A^{k}}=A^{k}\left(A^{G D}\right)^{k}$.

Lemma 2.8. [9, Lemma 3.7] Let $A, B \in \mathbb{C}^{n \times n}$. If $A X B=0$ for all $X \in \mathbb{C}^{n \times n}$, then $A=0$ or $B=0$.

REMARK 2.9. Let $P \in \mathbb{C}^{n \times n}$ be a nonsingular matrix. Let $A, B$ be square matrices of adequate sizes satisfying $M=P\left[\begin{array}{cc}A & 0 \\ 0 & B\end{array}\right] P^{-1}$. Then the following properties hold:

(i) If $A$ is nonsingular, then $\operatorname{ind}(M)=\operatorname{ind}(B)$,

(ii) $P\left[\begin{array}{cc}X & 0 \\ 0 & Y\end{array}\right] P^{-1} \in M\{G D\}$, for any $X \in A\{G D\}$ and $Y \in B\{G D\}$.

Theorem 2.10. Let $A, B \in \mathbb{C}^{n \times n}$ be such that $k=\operatorname{ind}(A), \ell=\operatorname{ind}(B), t=r k\left(B^{\ell}\right)$, satisfying $A \varliminf^{s} B$, and let $B^{G D}$ be a fixed $G$-Drazin inverse of $B$. Then, the following statements are equivalent:

(i) $B\{G D\} \subseteq A\{G D\}$ and $A\left(I-B^{G D} B\right) \neq 0$.

(ii) $A=R\left[\begin{array}{ccc}S_{1} & 0 & 0 \\ 0 & D S_{2} & D C_{2} \\ 0 & 0 & 0\end{array}\right] R^{-1}, B=R\left[\begin{array}{ccc}S_{1} & 0 & 0 \\ 0 & S_{2} & 0 \\ 0 & 0 & N\end{array}\right] R^{-1}$, where $R \in \mathbb{C}^{n \times n}, r=r k\left(A^{k}\right)$, $S_{1} \in \mathbb{C}^{r \times r}$, and $S_{2} \in \mathbb{C}^{(t-r) \times(t-r)}$ are nonsingular, $N \in \mathbb{C}^{(n-t) \times(n-t)}$ is nilpotent of index $\ell, D \in \mathbb{C}^{(t-r) \times(t-r)}$ is idempotent, and $C_{2} \in \mathbb{C}^{(t-r) \times(n-t)}$ such that $\left(D S_{2}\right)^{k-1} D=0$ and $D C_{2}\left(I_{n-t}-N^{-} N\right) \neq 0$. If $A$ is nilpotent $(r=0)$ then $S_{1}$ is absent.

Proof. $[($ ii $) \Rightarrow(\mathrm{i})]$ It is easy by definition.

$[(\mathrm{i}) \Rightarrow(\mathrm{ii})]$ Note that from Theorem 2.5 and (1.5) we have $k>1, \ell>0, t=\operatorname{rk}\left(B^{\ell}\right)>0$ and $t \neq \operatorname{rk}\left(A^{k}\right)$. By Theorem 2.2 (ii), we get $\operatorname{rk}\left(A^{k}\right)<t$. Using the projectors $P_{B}=B B^{G D}, Q_{B}=B^{G D} B$, and $P_{B^{\ell}}=$ $B^{\ell}\left(B^{G D}\right)^{\ell}$, from Theorem 2.7 it follows that

$$
X_{U, T}=B^{G D}+\left(I-P_{B^{\ell}}\right) U\left(I-P_{B}\right)+\left(I-Q_{B}\right) T\left(I-P_{B^{\ell}}\right)
$$


are all G-Drazin inverses of $B$, for arbitrary matrices $U$ and $T$. Since $X_{U, T}, B^{G D} \in B\{G D\}$, the equalities $A X_{U, T} A=A B^{G D} A=A$ hold. Then, setting $U=0$ in (2.10), it follows that $A\left(I-Q_{B}\right) T\left(I-P_{B^{\ell}}\right) A=0$, for arbitrary $T$. Therefore, from Lemma 2.8 we get $A\left(I-Q_{B}\right)=0$ or $\left(I-P_{B^{\ell}}\right) A=0$.

Since $A\left(I-Q_{B}\right) \neq 0$ by hypothesis, $\left(I-P_{B^{\ell}}\right) A=0$ must be true. Let

$$
B=P\left[\begin{array}{cc}
\Sigma & 0 \\
0 & N
\end{array}\right] P^{-1}
$$

be a core-nilpotent decomposition of $B$ where $P \in \mathbb{C}^{n \times n}, \Sigma \in \mathbb{C}^{t \times t}$ are both nonsingular, and $N \in$ $\mathbb{C}^{(n-t) \times(n-t)}$ is nilpotent of index $\ell$. From (1.3), we have

$$
B^{G D}=P\left[\begin{array}{cc}
\Sigma^{-1} & 0 \\
0 & N^{-}
\end{array}\right] P^{-1}, \quad \text { with } N^{-} \in N\{1\}
$$

Moreover,

$$
B^{\ell}=P\left[\begin{array}{cc}
\Sigma^{\ell} & 0 \\
0 & 0
\end{array}\right] P^{-1} \quad \text { and } \quad P_{B^{\ell}}=P\left[\begin{array}{cc}
I_{t} & 0 \\
0 & 0
\end{array}\right] P^{-1}
$$

We partition $A=P\left[\begin{array}{ll}A_{1} & A_{2} \\ A_{3} & A_{4}\end{array}\right] P^{-1}$ accordingly to the sizes of the blocks of $B$. Since $\left(I-P_{B^{\ell}}\right) A=0$, it follows that $A_{3}=0$ and $A_{4}=0$, then

$$
A=P\left[\begin{array}{cc}
A_{1} & A_{2} \\
0 & 0
\end{array}\right] P^{-1}
$$

Some easy computations show that $B\{G D\} \subseteq A\{G D\}$ implies $\left\{\Sigma^{-1}\right\}=\Sigma\{G D\} \subseteq A_{1}\{G D\} \subseteq A_{1}\{1\}$ and $A_{1} \Sigma^{-1} A_{2}=A_{2}$. Now, $A_{1} \leq^{-} \Sigma$. Note that $A_{1} \neq 0$. Otherwise, $A_{2}=0$ and so $A=0$, which contradicts the fact that $A\left(I-Q_{B}\right) \neq 0$. Now, let

$$
A_{1}=Q\left[\begin{array}{cc}
S_{1} & 0 \\
0 & M
\end{array}\right] Q^{-1}
$$

be the core-nilpotent decomposition of $A_{1}$, where $Q \in \mathbb{C}^{t \times t}$ and $S_{1} \in \mathbb{C}^{r \times r}$ are both nonsingular matrices, and $M \in \mathbb{C}^{(t-r) \times(t-r)}$ is nilpotent of index $m=\operatorname{ind}\left(A_{1}\right)$.

According to [16, Theorem 3.1] we have $\Sigma=Q\left[\begin{array}{cc}S_{1} & 0 \\ 0 & S_{2}\end{array}\right] Q^{-1}$, with $M \leq^{-} S_{2}$. Notice that $S_{2} \in$ $\mathbb{C}^{(t-r) \times(t-r)}$ is nonsingular. Now, we consider

$$
R=P\left[\begin{array}{cc}
Q & 0 \\
0 & I_{n-t}
\end{array}\right] \in \mathbb{C}^{n \times n} \text { and } Q^{-1} A_{2}=\left[\begin{array}{l}
C_{1} \\
C_{2}
\end{array}\right] \in \mathbb{C}^{t \times(n-t)},
$$

where $C_{1} \in \mathbb{C}^{r \times(n-t)}$ and $C_{2} \in \mathbb{C}^{(t-r) \times(n-t)}$. Easy computations show that

$$
\begin{gathered}
A=R\left[\begin{array}{ccc}
S_{1} & 0 & C_{1} \\
0 & M & C_{2} \\
0 & 0 & 0
\end{array}\right] R^{-1}, \quad B=R\left[\begin{array}{ccc}
S_{1} & 0 & 0 \\
0 & S_{2} & 0 \\
0 & 0 & N
\end{array}\right] R^{-1} \\
A^{k}=R\left[\begin{array}{ccc}
S_{1}^{k} & 0 & S_{1}^{k-1} C_{1} \\
0 & M^{k} & M^{k-1} C_{2} \\
0 & 0 & 0
\end{array}\right] R^{-1}, \quad \text { and } \quad B^{\ell}=R\left[\begin{array}{ccc}
S_{1}^{\ell} & 0 & 0 \\
0 & S_{2}^{\ell} & 0 \\
0 & 0 & 0
\end{array}\right] R^{-1} .
\end{gathered}
$$


On the other hand, from Lemma 2.3 we get $A^{k} \preceq^{s} B^{\ell}$. Thus, [13, Theorem 3.2.10] ensures that $A^{k}=$ $R\left[\begin{array}{ll}Z & 0 \\ 0 & 0\end{array}\right] R^{-1}$, with $Z \in \mathbb{C}^{t \times t}$ and $\operatorname{ind}(Z) \leq 1$. Then, $S_{1}^{k-1} C_{1}=0$ and $M^{k-1} C_{2}=0$, which implies $C_{1}=0$ because $S_{1}$ is nonsingular.

Moreover, $A_{1} \Sigma^{-1} A_{2}=A_{2}$ yields

$$
M S_{2}^{-1} C_{2}=C_{2} \text {. }
$$

Setting $W:=\left[\begin{array}{cc}M & C_{2} \\ 0 & 0\end{array}\right]$ we have that $W^{j+1}=\left[\begin{array}{cc}M^{j+1} & M^{j} C_{2} \\ 0 & 0\end{array}\right]=0$, for $j:=\max \{k, m\}$. That is, $W$ is a nilpotent matrix. From (2.11), we obtain

$$
A=R\left[\begin{array}{cc}
S_{1} & 0 \\
0 & W
\end{array}\right] R^{-1}
$$

By Remark 2.9, we get $i n d(W)=\operatorname{ind}(A)=k$ due to nonsingularity of $S_{1}$. Consequently, (2.13) gives the core-nilpotent decomposition of $A$. Then, $\operatorname{ind}(M)=k$, and hence,

$$
M^{k}=0 .
$$

Since $M \leq^{-} S_{2}$ and $\left(I_{t-r}, S_{2}\right)$ is a full-rank factorization of $S_{2}$, [13, Theorem 3.3.5] implies that there exists an idempotent matrix $D \in \mathbb{C}^{(t-r) \times(t-r)}$ such that $M=D S_{2}$. Hence, from (2.12) and (2.14) it follows that $C_{2}=D C_{2}$ and $\left(D S_{2}\right)^{k-1} D=0$, respectively. Finally, as

$$
A\left(I-Q_{B}\right)=R\left[\begin{array}{ccc}
0 & 0 & 0 \\
0 & 0 & D C_{2}\left(I_{n-t}-N^{-} N\right) \\
0 & 0 & 0
\end{array}\right] R^{-1}
$$

it then follows that (ii) holds.

Similarly, we can obtain the following result by using (1.4) and the above theorem.

Theorem 2.11. Let $A, B \in \mathbb{C}^{n \times n}$ be such that $k=\operatorname{ind}(A), \ell=\operatorname{ind}(B), t=r k\left(B^{\ell}\right)$, satisfying $A \swarrow^{s} B$, and let $B^{G D}$ be a fixed $G$-Drazin inverse of $B$. Then, the following statements are equivalent:

(i) $B\{G D\} \subseteq A\{G D\}$ and $\left(I-B B^{G D}\right) A \neq 0$.

(ii) $A=R\left[\begin{array}{ccc}S_{1} & 0 & 0 \\ 0 & S_{2} D & 0 \\ 0 & C_{2} D & 0\end{array}\right] R^{-1}, B=R\left[\begin{array}{ccc}S_{1} & 0 & 0 \\ 0 & S_{2} & 0 \\ 0 & 0 & N\end{array}\right] R^{-1}$, where $R \in \mathbb{C}^{n \times n}, r=r k\left(A^{k}\right), S_{1} \in$ $\mathbb{C}^{r \times r}$, and $S_{2} \in \mathbb{C}^{(t-r) \times(t-r)}$ are nonsingular, $N \in \mathbb{C}^{(n-t) \times(n-t)}$ is nilpotent of index $\ell, D \in \mathbb{C}^{(t-r) \times(t-r)}$ is idempotent, and $C_{2} \in \mathbb{C}^{(n-t) \times(t-r)}$, such that $D\left(S_{2} D\right)^{k-1}=0$ and $\left(I_{n-t}-N N^{-}\right) C_{2} D \neq 0$. Again, if $A$ is nilpotent then $S_{1}$ must be absent.

3. Algorithm and numerical examples. It is easy to check that the condition $A \preceq^{s} B$ in Theorem 2.4 is redundant in item (ii) for $n=1,2$. Next, for any given size $n \geq 3$ of the involved matrices, we provide an algorithm to construct two matrices $A$ and $B$ such that $B\{G D\} \subseteq A\{G D\}$ and $A \not^{G D} B$.

The expression $e_{j}$ denotes the canonical basis vector in $\mathbb{C}^{t \times 1}$ with 1 in the position $j$-th and 0 's otherwise. In addition, the notation $J(1: d-1,1: d)$ means to select the $d-1$ first rows and the $d$ first columns in the matrix $J$. 


\section{Algorithm}

Input: An integer $n \geq 3$.

Output: $A, B \in \mathbb{C}^{n \times n}$ such that $B\{G D\} \subseteq A\{G D\}$ and $A \mathbb{E}^{G D} B$.

Step 1. Set an integer $t$ such that $2 \leq t \leq n-1$.

Step 2. Set an integer $d$ such that $1 \leq d<t$ and $D=\operatorname{diag}\left(I_{d}, 0_{(t-d) \times(t-d)}\right)$.

Step 3. If $d \geq 2$, then construct $J=\left[\begin{array}{cc}0_{(d-1) \times 1} & I_{d-1} \\ 0 & 0_{1 \times(d-1)}\end{array}\right]$.

Step 4. If $d=1$, then set

$$
S=\left[\begin{array}{c}
e_{t}^{T} \\
e_{t-1}^{T} \\
\vdots \\
e_{2}^{T} \\
e_{1}^{T}
\end{array}\right]
$$

else set

$$
S=\left[\begin{array}{cc}
J(1: d-1,1: d) & 0_{(d-1) \times(t-d)} \\
\hline e_{t}^{T} & e_{t-1}^{T} \\
\vdots \\
e_{d+1}^{T} \\
e_{1}^{T}
\end{array}\right] \in \mathbb{C}^{t \times t} .
$$

Step 5. Choose $F \in \mathbb{C}^{d \times(n-t)}$ with entry equals 1 in position $(1,1)$ and 0 's elsewhere and set

$$
C=\left[\begin{array}{c}
F \\
0_{(t-d) \times(n-t)}
\end{array}\right] \text {. }
$$

Step 6. Construct $A=\left[\begin{array}{cc}D S & D C \\ 0_{(n-t) \times t} & 0_{(n-t) \times(n-t)}\end{array}\right]$ and $B=\left[\begin{array}{cc}S & 0 \\ 0 & 0_{(n-t) \times(n-t)}\end{array}\right]$.

\section{End}

We proceed to justify the Algorithm. We must discard all the cases for which Theorem 2.5 ensures that the implication $B\{G D\} \subseteq A\{G D\}$ implies $A \leq G D B$ holds. Moreover, we construct a nilpotent matrix $A$ and an index 1 matrix $B$. For matrices $A$ and $B$ in Step 6, it is easy to check that: $A$ has index $d+1$ and, by definition, $B\{G D\} \subseteq A\{G D\}$. The last point is to see that $A \not^{G D} B$. In order to do that, we can compute $A\left(I-B B^{-}\right)$which does not vanishes for $B^{-}=\left[\begin{array}{cc}S^{-1} & 0 \\ 0 & 0\end{array}\right]$.

Example 3.1. Consider $n=3$. From Steps 1 to 4 , the Algorithm gives $t=2, d=1$, that is

$$
D=\left[\begin{array}{ll}
1 & 0 \\
0 & 0
\end{array}\right] \text { and } S=\left[\begin{array}{ll}
0 & 1 \\
1 & 0
\end{array}\right] \text {. }
$$


Step 5 provides $C=\left[\begin{array}{l}1 \\ 0\end{array}\right]$. From Step 6 , we conclude that

$$
A=\left[\begin{array}{lll}
0 & 1 & 1 \\
0 & 0 & 0 \\
0 & 0 & 0
\end{array}\right] \text { and } B=\left[\begin{array}{ccc}
0 & 1 & 0 \\
1 & 0 & 0 \\
0 & 0 & 0
\end{array}\right]
$$

satisfy $B\{G D\} \subseteq A\{G D\}$ and $A \not^{G D} B$.

ExAmple 3.2. Consider $n=7$. In contrast to Example 3.1, Steps 1 to 2 from Algorithm does not give only one possibility. Thus, we choose $t=5$ and $d=3$. It also provides $D=\operatorname{diag}\left(I_{3}, 0_{2}\right)$. Step 3 allows us to construct

$$
J=\left[\begin{array}{cc}
0_{2 \times 1} & I_{2} \\
0 & 0_{1 \times 2}
\end{array}\right]
$$

and, from Steps 4 and 5, we obtain

$$
S=\left[\begin{array}{lllll}
0 & 1 & 0 & 0 & 0 \\
0 & 0 & 1 & 0 & 0 \\
0 & 0 & 0 & 0 & 1 \\
0 & 0 & 0 & 1 & 0 \\
1 & 0 & 0 & 0 & 0
\end{array}\right] \text { and } C=\left[\begin{array}{cc}
1 & 0 \\
0 & 0 \\
0 & 0 \\
0 & 0 \\
0 & 0
\end{array}\right] .
$$

From Step 6, we conclude that

$$
A=\left[\begin{array}{lllllll}
0 & 1 & 0 & 0 & 0 & 1 & 0 \\
0 & 0 & 1 & 0 & 0 & 0 & 0 \\
0 & 0 & 0 & 0 & 1 & 0 & 0 \\
0 & 0 & 0 & 0 & 0 & 0 & 0 \\
0 & 0 & 0 & 0 & 0 & 0 & 0 \\
0 & 0 & 0 & 0 & 0 & 0 & 0 \\
0 & 0 & 0 & 0 & 0 & 0 & 0
\end{array}\right] \text { and } B=\left[\begin{array}{ccccccc}
0 & 1 & 0 & 0 & 0 & 0 & 0 \\
0 & 0 & 1 & 0 & 0 & 0 & 0 \\
0 & 0 & 0 & 0 & 1 & 0 & 0 \\
0 & 0 & 0 & 1 & 0 & 0 & 0 \\
1 & 0 & 0 & 0 & 0 & 0 & 0 \\
0 & 0 & 0 & 0 & 0 & 0 & 0 \\
0 & 0 & 0 & 0 & 0 & 0 & 0
\end{array}\right] .
$$

Acknowledgements. We would like to thank the referees for their valuable comments and suggestions which helped us to improve the presentation of the paper.

\section{REFERENCES}

[1] J.K. Baksalary, J. Hauke, and G.P.H. Styan. On some distributional properties of quadratic forms in normal variables and some associated matrix partial orderings. Multivariate Analysis and its Applications, 24:111-121, 1994.

[2] S.L. Campbell. Recent Applications of Generalized Inverses. Pitman, London, 1982.

[3] S.L. Campbell and C.D. Meyer. Generalized Inverses of Linear Transformations. SIAM, Philadelphia, 2009.

[4] S.L. Campbell and C.D. Meyer. Weak Drazin inverses. Linear Algebra and its Applications, 20:167-178, 1978.

[5] C. Coll, M. Lattanzi, and N. Thome. Weighted G-Drazin inverses and a new pre-order on rectangular matrices. Applied Mathematics and Computation, 317:12-24, 2018.

[6] D.S. Cvetković-Ilić, D. Mosić, and Y. Wei. Partial orders on B(H). Linear Algebra and its Applications, 481:115-130, 2015.

[7] M.S. Djikić. Lattice properties of the core-partial order. Banach Journal of Mathematical Analysis, 11:398-415, 2017. 
[8] K.L. Doty, C. Melchiorri, and C. Bonivento. A Theory of Generalized Inverses Applied to Robotics. The International Journal of Robotics Research, 12:1-19, 1993.

[9] D.E. Ferreyra, M. Lattanzi, F.E. Levis, and N. Thome. Parametrized solutions $X$ of the system $A X A=A E A$ and $A^{k} E A X=X A E A^{k}$ for a matrix $A$ having index $k$. Electronic Journal of Linear Algebra, 35:503-510, 2019.

[10] M.I. Gareis, M. Lattanzi, and N. Thome. Nilpotent matrices and the minus partial order. Quaestiones Mathematicae, 40:519-525, 2017.

[11] J. Mielniczuk. Note on the core matrix partial ordering. Discussiones Mathematicae, Probability and Statistics, 31:71-75, 2011.

[12] S.K. Mitra. On group inverses and the sharp order. Linear Algebra and its Applications, 92:17-37, 1987.

[13] S.K. Mitra, P. Bhimasankaram, and S. Malik. Matrix Partial Orders, Shorted Operators and Applications. World Scientific Publishing Company, Singapore, 2010.

[14] F. Soleimani, P.S. Stanimirović, and F. Soleymani. Some matrix iterations for computing generalized inverses and balancing chemical equations. Algorithms, 8:982-998, 2015.

[15] M. Tosić and D.S. Cvetković-Ilić. Invertibility of a linear combination of two matrices and partial orderings. Applied Mathematics and Computation, 218:4651-4657, 2012.

[16] X. Wang and X. Liu. Partial orders based on core-nilpotent decomposition. Linear Algebra and its Applications, 488:235248, 2016.

[17] Y. Wei, P. Stanimirović, and M. Petković. Numerical and Symbolic Computations of Generalized Inverses. World Scientific, Singapore, 2018.

[18] G.Z. Xiao, B.Z. Shen, C.K. Wu, and C.S. Wong. Some spectral techniques in coding theory. Discrete Mathematics, 87:181-186, 1991. 